\title{
Effect of exotic tree plantation on floristic composition and phytodiversity of Rema-Kalenga wildlife sanctuary, Bangladesh
}

\author{
Gazi Mosharof Hossain* and A.B.M. Enayet Hossain \\ Department of Botany, Faculty of Biological Sciences, Jahangirnagar University, Savar, \\ Dhaka-1342, Bangladesh
}

\begin{abstract}
Effect of exotic tree plantation on floristic composition and phytodiversity status of Rema-Kalenga wildlife sanctuary of Bangladesh was studied. A total of 309 vascular plant species under 245 genera belonging to 83 families were found to constitute the vascular flora of the studied area. The maximum number of species (298) with the highest Shannon-Weiner diversity index value (3.882 \pm 0.090$)$ was recorded from natural forest, which was followed by 194 and 165 plant species with $3.441 \pm 0.205$ and $3.398 \pm 0.103$ diversity index values recorded from Tectona and Acacia plantation sites respectively. The minimum number of plant species (142) with the lowest diversity index value (2.999 \pm 0.152$)$ was recorded from Eucalyptus plantation site. The collected data on the selected forest sites of Rema-Kalenga wildlife sanctuary showed the trends of gradual decrease in floristic composition and phytodivesity status of three plantation sites (Tectona to Acacia to Eucalyptus) in respect to natural forest, which indicated that exotic tree plantations might have negative impact on floristic composition and phytodiversity of this semi-tropical forest area and the fast-growing exotic tree plantation of Acacia and Eucalyptus should be avoided for sustainable development of Rema-Kalenga wildlife sanctuary.
\end{abstract}

Key words: Fast-growing exotic tree plantation, floristic composition, phytodiversity, Rema-Kalenga wildlife sanctuary, Bangladesh.

\section{INTRODUCTION}

The global extent of plantation forests in 1990 is estimated to be around 135 million ha (FAO, 1993; Gauthier, 1991; Pandy, 1995). About 75\% of these plantation forests are in temperate regions and about $25 \%$ in the tropics and subtropics; some $5 \%$ are found in Africa, a little more than $10 \%$ in each of the American continents, some $20 \%$ in the former USSR and around 25\% in each of Asia-Pacific and Europe (Gauthier, 1991; Kanowski \& Savill, 1992). Most plantation forests have been established as even-aged monoculture crops of tree species with the primary purpose of wood production (Evans, 1992). Around $90 \%$ of existing plantations have been established for the production of wood for industrial use and most of the remainder to produce wood for use as fuel or round wood. Some plantation forests are grown and managed, either primarily or jointly, for non-wood products such as betel leaf, medicinal plants or fodder. Fast-growing tree plantations, mainly Acacia and Eucalyptus are widespread in tropical countries (Bouillet \& Bernhard-Reversat, 2001). In the 19th and 20th Centuries valuable timber species and fast growing tree species of Australian origin were introduced to facilitate the development of forestry in Bangladesh (Hossain \& Pasha, 2001; Islam et al., 2003; Hossain, 2006). Last few decades some fast-growing exotic tree species like akashmoni (Acacia auriculiformis A. Cunn. ex. Benth.) and eucalyptus (Eucalyptus camaldulensis

* Corresponding author Email: gazibotju@gmail.com 
Hook.) have been planted in different areas of Bangladesh. Most of the original forest cover of Rema-Kalenga wildlife sanctuary has been removed or substantially altered through plantation programs or other anthropogenic activities. Plantation started in this region from 1922 and in early stage, the Forest Department started the plantation program by planting teak tree (Tectona grandis L.). Then they took several initiatives to extend natural forest in degraded and deforested areas. As a consequence they planted some fastgrowing exotic tree species like Acacia and Eucalyptus in some areas of the sanctuary. Environmental impact of fast-growing tree plantations in the tropics has been a controversial topic. Some authors argued that these species exhaust soil water and nutrient resources, and prevent undergrowth vegetation and in further soil erosion and loss of fertility (Poore \& Fries, 1985; Abbasi \& Vinithan, 1997; Bouvet, 1998). The fast-growing exotic tree plantations are not suitable for local undergrowth vegetation (Hossain et al., 2002) as well as for soil nutritional quality (Prasad, 1988) and microbial growth (Hossain and Hossain, 2009). The previous studies conducted on this sanctuary were on taxonomy, ethnobotany, soil ecology etc. (Uddin et al., 2001; Uddin \& Hassan, 2004; Hossain \& Hossain, 2009). Data on the effects of different plantations on the vegetation in this area is still lacking. Therefore, the present investigation in this sanctuary was conducted to know the impacts of plantations of exotic tree species on the floristic composition and phytodiversity status of this area and to provide data that might be helpful for proper management plan and sustainable development of the sanctuary.

\section{MATERIALS AND METHODS}

The Rema-Kalenga wildlife sanctuary is located at Chunarughat thana of Habigonj district under the forest administration unit of Habigonj-2 forest range of Sylhet forest division (Fig. 1). Geographically, the study area is situated between $24^{\circ} 06^{\prime}$ to $24^{\circ} 14^{\prime} \mathrm{N}$ latitude and $91^{\circ} 34^{\prime}$ to $91^{\circ} 41^{\prime} \mathrm{E}$ longitude with about $67 \mathrm{~m}$ elevation at the highest peak (Rizvi, 1970). In 1982, this area was declared as a wildlife sanctuary through a Gazette Notification No. 11/For-68/89/882, Date $22 / 12 / 1981$ by the Government under the Bangladesh Wildlife (Preservation) Order, 1973 (Amendment Act 1974) to provide for the preservation, conservation and management of wildlife in Bangladesh. The sanctuary falls under IUCN management category IV- habitat/species management area (Green, 1990). This area is under the bio-ecological zone-9b with broad zone 'Sylhet Hills' (Nishat et. al., 2002). According to Bangladesh agro- ecological

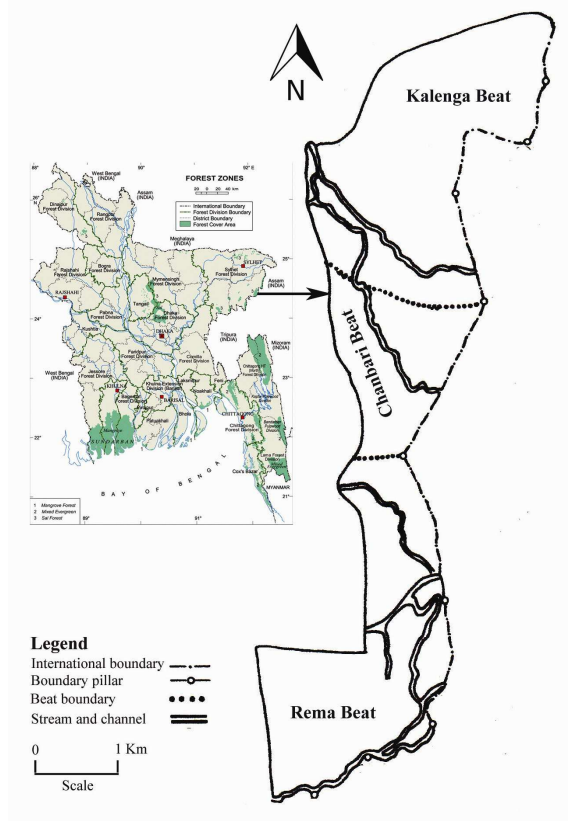

Fig. 1. Map of the Rema-Kalenga wildlife sanctuary. 
zones, this area belongs to Region-29, 'Northern and Eastern Hills', sub-region-29c, 'Low hills and piedmont plains' (FAO, 1988). Current notified area of this sanctuary is 1795.54 ha. (Canonizado \& Rahman, 1998). This forest area covers an area of low-medium high hills formed primarily from soft sandstones and classified as a mixed tropical evergreen forest (Champion, 1936). Sub-tropical monsoon climate exists in the study area with three distinct seasons, viz. summer, monsoon and winter. The mean minimum and maximum temperatures varied between $11.8^{\circ} \mathrm{C}$ and $33.2^{\circ} \mathrm{C}$, relative humidity ranged from $71.5 \%$ to $87.5 \%$ and mean annual rainfall varied from 7.3 to $456.8 \mathrm{~mm}$ (Uddin and Hassan, 2004). The relief is characterized by a gently undulating to hilly topography and the soils are mainly sandy loam to silt clay with acidic in nature (Hossain, 2006).

Sample collection and analysis: Plant samples were collected from four different types of standing forest sites, viz., natural forest, teak (Tectona grandis L.), acacia (Acacia auriculiformis A. Cunn. ex. Benth.) and eucalyptus (Eucalyptus camaldulensis Hook.) plantation forests sites during winter, summer and monsoon seasons from 2003 to 2005. Undergrowth plant samples of the selected sites were collected by standard quadrat method (Braun-Blanquet, 1932; Raunkiaer, 1934) and the quadrat size was determined as $10 \mathrm{~m} \times 10 \mathrm{~m}$ following species-area curve method (Braun-Blanquet, 1932). Collected plant specimens were properly processed using standard herbarium techniques (Hyland, 1972; Jain \& Rao, 1977 and Alexiades, 1996). The specimens were identified through consulting with expert taxonomists, cross-checking with herbarium specimens preserved at JUH and Bangladesh National Herbarium (DACB); and also matching with different relevant taxonomic literatures, viz., Hooker (1872-1897), Prain (1903), Uddin and Hassan (2004), Siddiqui et al. (2007) and Ahmed et al. (2008-2009).

Shannon-Wiener diversity index of the recorded undergrowth plants of different selected sites was calculated through the following formula described as Kent and Coker (1992).

Shannon-Wiener Diversity Index $\left(\mathrm{H}^{\prime}\right)=-\Sigma$ pi ln pi

where,

$\mathrm{pi}=$ The proportion of individuals or the abundance of the

ith species expressed as a proportion of total cover,

$\ln =$ Log $_{\text {base }_{\mathrm{n}}}$

\section{RESULTS AND DISCUSSION}

The collected data on the floristic composition and Shannon-Wiener diversity index of the undergrowth plant species of selected forest sites of Rema-Kalenga wildlife sanctuary have been presented in Tables 1 to 2 and Figs. 2 to 5. In this study, a total of 309 vascular plant species under 245 genera belonging to 83 families were identified as undergrowth species (Table 1). The maximum number of species $(250 ; 80.91 \%)$ belonged to dicotyledons and it was followed by $47(15.21 \%)$ and $12(3.88 \%)$ species, respectively of monocotyledons and pteridophytes (Fig. 3). The highest number of species 100 (32.36\%) were recorded as herb, which was followed by $93(30.10 \%), 71(22.98 \%)$ and 45 $(14.56 \%)$ species, respectively of tree, shrub and climber respectively (Fig. 4). 
In natural forest, a total of 304 plant species under 240 genera and 81 families were found (Fig. 2), of which 298 species under 237 genera of 79 families were recorded during monsoon season, 287 species under 234 genera of 75 families during winter season, whereas 245 species under 221 genera belonging to 69 families in summer season (Table 2). The values of Shannon-Wiener diversity index of the recorded undergrowth species of natural forest site varied between 3.960 and 3.784. The highest index value 3.960 was calculated during monsoon season which was followed by 3.901 and 3.784 recorded during winter and summer seasons, respectively (Fig. 5).

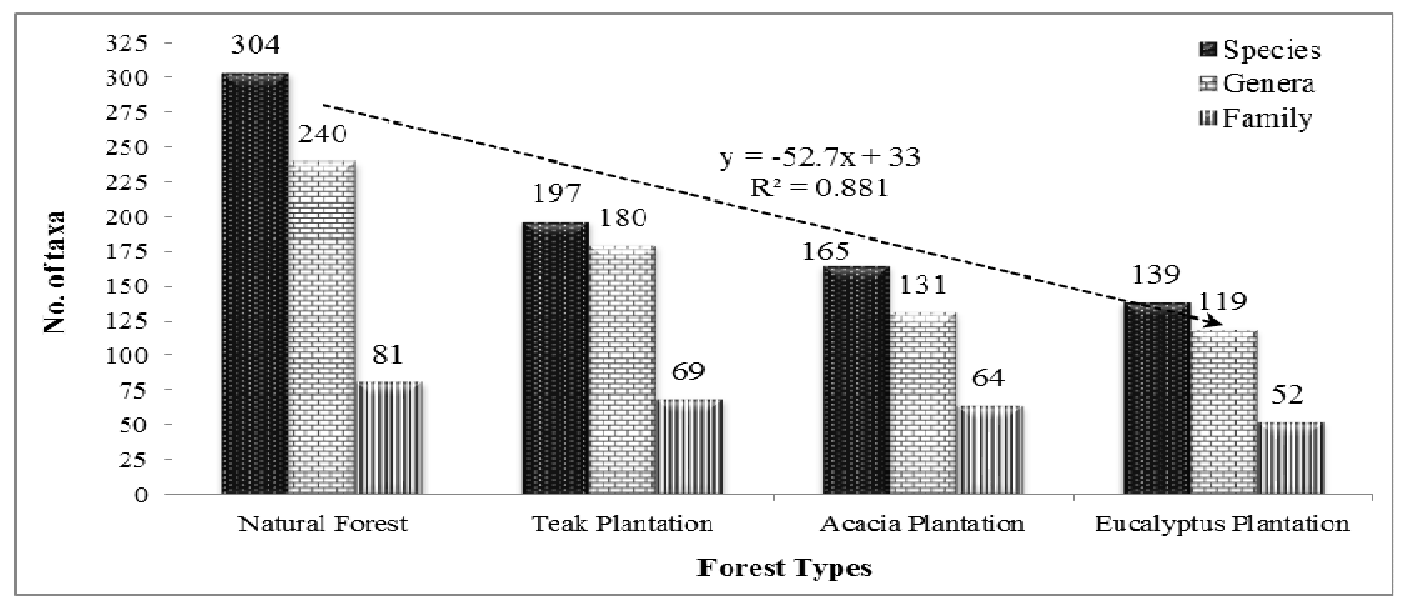

Fig. 2. Floristic composition of undergrowth plant species recorded from four types of forest sites of Rema-Kalenga wildlife sanctuary

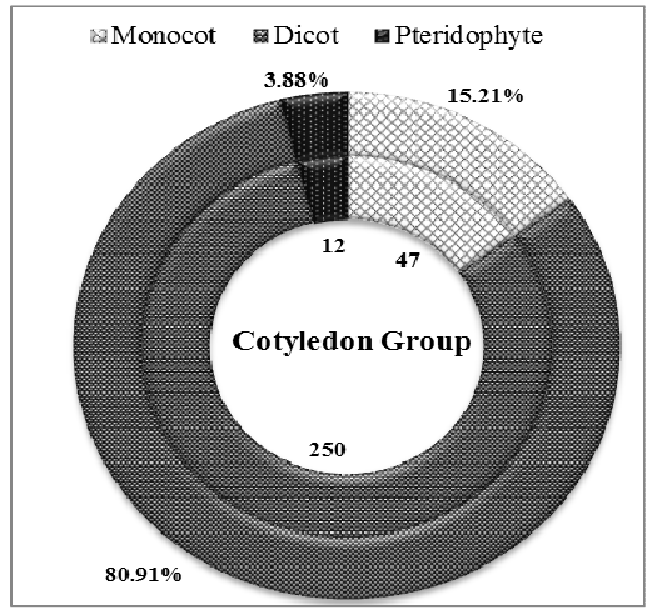

Fig. 3. Major groups of undergrowth plant species recorded from four types of forest sites of Rema-Kalenga wildlife sanctuary

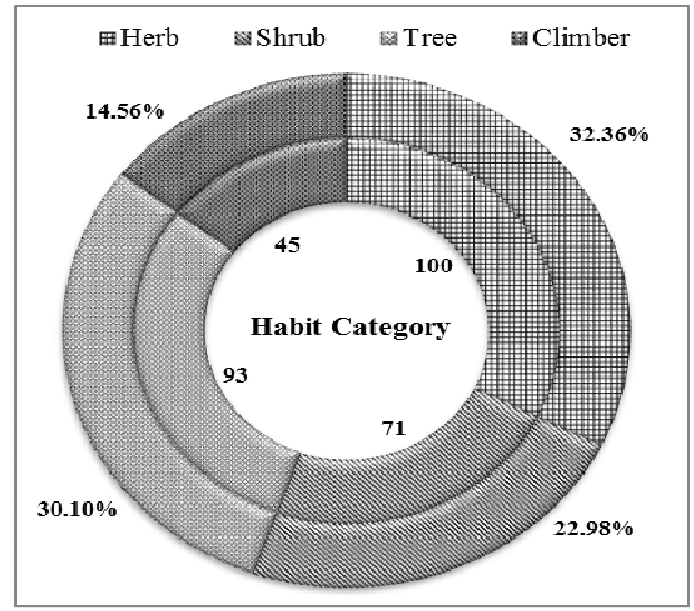

Fig. 4. Habit categories of undergrowth plant species recorded from four types of forest sites of Rema-Kalenga wildlife sanctuary 
Table 1. Comprehensive checklist of vascular plant species recorded from four different types of forest sites of Rema-Kalenga wildlife sanctuary

\begin{tabular}{|c|c|c|c|c|}
\hline Sl. No. & Scientific name & Family & Cotyledon & Habit \\
\hline 1. & Abelmoschus moschatus Medic. & Malvaceae & Dicot & Shrub \\
\hline 2. & Abutilon indicum (L.) Sweet & Malvaceae & Dicot & Shrub \\
\hline 3. & Abrus precatorius $\mathrm{L}$. & Fabaceae & Dicot & Climber \\
\hline 4. & Acacia auriculiformis A. Cunn. ex Benth. \& Hook. & Fabaceae & Dicot & Tree \\
\hline 5. & Acacia mangium Willd. & Fabaceae & Dicot & Tree \\
\hline 6. & Acacia nilotica (L.) Delile & Fabaceae & Dicot & Tree \\
\hline 7. & Achyranthus aspera $\mathrm{L}$. & Acanthaceae & Dicot & Herb \\
\hline 8. & Adhatoda zeylanica Medikus & Acanthaceae & Dicot & Shrub \\
\hline 9. & Adiantum phillippense $\mathrm{L}$. & Adiantaceae & Pteridophyte & Herb \\
\hline 10. & Aegle marmelos (L.) Corr. & Rutaceae & Dicot & Tree \\
\hline 11. & Ageratum conyzoides $\mathrm{L}$. & Asteraceae & Dicot & Herb \\
\hline 12. & Aglaonema hookerianum Schott. & Araceae & Monocot & Herb \\
\hline 13. & Albizia chinensis (Osb.) Merr. & Fabaceae & Dicot & Herb \\
\hline 14. & Albizia lebbeck (L.) Benth. \& Hook. & Fabaceae & Dicot & Herb \\
\hline 15. & Albizia odorattisima (L. f.) Benth. & Fabaceae & Dicot & Herb \\
\hline 16. & Albizia procera (Roxb.) Benth. & Fabaceae & Dicot & Herb \\
\hline 17. & Aleuritopteris grisea (Blanford) Panigrahi & Sinopteridaceae & Pteridophyte & Herb \\
\hline 18. & Allophylus cobbe (L.) Raeuschel & Sapindaceae & Dicot & Shrub \\
\hline 19. & Alocasia auminata Schott. & Araceae & Monocot & Herb \\
\hline 20. & Alpinia allughas (Retz.) Rose. & Zingiberaceae & Monocot & Herb \\
\hline 21. & Alpinia galangal $(\mathrm{L}.) \mathrm{Sw}$. & Zingiberaceae & Monocot & Herb \\
\hline 22. & Alpinia malaceensis (Burm. f.) Rose. & Zingiberaceae & Monocot & Herb \\
\hline 23. & Alstonia scholaris (L.) R. Br. & Apocynaceae & Dicot & Tree \\
\hline 24. & Alternanthera sesilis (L.) R. Br. ex Roem. \& Schult. & Amaranthaceae & Dicot & Herb \\
\hline 25. & Amaranthus spinosus L. & Amaranthaceae & Dicot & Herb \\
\hline 26. & Amorphophallus bulbifer (Roxb.) Blume & Araceae & Monocot & Herb \\
\hline 27. & Ampelopteris prolifera (Retz.) Copel. & Thelypteridaceae & Pteridophyte & Herb \\
\hline 28. & Ampelocissus latifolia (Roxb.) Planch. & Vitaceae & Dicot & Climber \\
\hline 29. & Angiopteris evecta (Forst) Hoffm. & Angiopteridaceae & Pteridophyte & Herb \\
\hline 30. & Anisomeles ovata $\mathrm{R} . \mathrm{Br}$. & Lamiaceae & Dicot & Herb \\
\hline 31. & Anona reticulata $\mathrm{L}$. & Annonaceae & Dicot & Shrub \\
\hline 32. & Anthocephalus chinensis (Lamk.) A. Rich. ex Walp. & Rubiaceae & Dicot & Tree \\
\hline 33. & Antidesma bunius (L.) Spreng. & Euphorbiaceae & Dicot & Shrub \\
\hline 34. & Antidesma ghaesembilla Gaertn. & Euphorbiaceae & Dicot & Shrub \\
\hline 35. & Aphanamixis polystachya (Wall.) R. N. Parker & Meliaceae & Dicot & Tree \\
\hline 36. & Aquilaria agallocha Roxb. & Thymeliaceae & Dicot & Tree \\
\hline 37. & Aporosa dioica (Roxb.) Muell.-Arg. & Euphorbiaceae & Dicot & Shrub \\
\hline 38. & Ardisia solanacea (Poir.) Roxb. & Myrsinaceae & Dicot & Shrub \\
\hline 39. & Argyreia nervosa (Burm. f.) Boj. & Convolvulaceae & Dicot & Climber \\
\hline 40. & Aristolochia tagal Cham. & Aristolochiaceae & Dicot & Climber \\
\hline 41. & Artocarpus chaplasha Roxb. & Moraceae & Dicot & Tree \\
\hline 42. & Artocarpus heterophyllus Lamk. & Moraceae & Dicot & Tree \\
\hline 43. & Artocarpus lacucha Buch.-Ham. & Moraceae & Dicot & Tree \\
\hline 44. & Atylosia scarabaeoides (L.) Baker & Fabaceae & Dicot & Climber \\
\hline 45. & Averrhoea carambola $\mathrm{L}$. & Averrhoaceae & Dicot & Tree \\
\hline 46. & Axonopus compressus (Sw.) P. Beauv. & Poaceae & Monoot & Herb \\
\hline 47. & Azadirachta indica A. Juss. & Meliaceae & Dicot & Tree \\
\hline 48. & Baccaurea ramiflora Lour. & Euphorbiaceae & Dicot & Tree \\
\hline 49. & Bambusa tulda Roxb. & Poaceae & Monocot & Tree \\
\hline
\end{tabular}


Table 1. Continued...

\begin{tabular}{|c|c|c|c|c|}
\hline Sl. No. & Scientific name & Family & Cotyledon & Habit \\
\hline 50. & Bauhinia purpurea $\mathrm{L}$. & Fabaceae & Dicot & Tree \\
\hline 51. & Bauhinia variegata $\mathrm{L}$. & Fabaceae & Dicot & Tree \\
\hline 52. & Blumea lacera (Burm. f.) DC. & Asteraceae & Dicot & Herb \\
\hline 53. & Boerhaavia diffusa $\mathrm{L}$. & Nyctaginaceae & Dicot & Herb \\
\hline 54. & Bombax ceiba Burm. f. & Bombacaceae & Dicot & Tree \\
\hline 55. & Borreria articularis (L. f.) Williams & Rubiaceae & Dicot & Herb \\
\hline 56. & Breynia retusa (Dennst.) Alston & Euphorbiaceae & Dicot & Shrub \\
\hline 57. & Bridelia stipularis (L.) Blume & Euphorbiaceae & Dicot & Shrub \\
\hline 58. & Buettneria pilosa Roxb. & Sterculiaceae & Dicot & Climber \\
\hline 59. & Butea monosperma (Lamk.) Taub. & Fabaceae & Dicot & Tree \\
\hline 60. & Caesalpinia bonduc (L.) Roxb. & Fabaceae & Dicot & Shrub \\
\hline 61. & Calamus guruba Buch.-Ham. ex Martius & Arecaceae & Monocot & Climber \\
\hline 62. & Calamus tenuis Roxb. & Arecaceae & Monocot & Climber \\
\hline 63. & Calamus viminalis Willd. & Arecaceae & Monocot & Climber \\
\hline 64. & Callicarpa longifolia Lamk. & Verbinaceae & Dicot & Shrub \\
\hline 65. & Calotropis procera (Ait.) R. Br. & Asclepiadaceae & Dicot & Shrub \\
\hline 66. & Carex jackiana Boott & Cyperaeae & Monocot & Herb \\
\hline 67. & Careya arborea Roxb. & Lecythidaceae & Dicot & Tree \\
\hline 68. & Caryota urens $\mathrm{L}$. & Arecaceae & Monocot & Tree \\
\hline 69. & Cassia fistula $\mathrm{L}$. & Fabaceae & Dicot & Tree \\
\hline 70. & Caturnaregam spinosa (Thumb.) Tirveng. & Rubiaceae & Dicot & Shrub \\
\hline 71. & Senna occidentalis Roxb. & Fabaceae & Dicot & Shrub \\
\hline 72. & Cassia siamea Lamk. & Fabaceae & Dicot & Tree \\
\hline 73. & Senna sophera (L.) Roxb. & Fabaceae & Dicot & Shrub \\
\hline 74. & Senna tora (L.) Roxb. & Fabaceae & Dicot & Herb \\
\hline 75. & Castanopsis tribuloides (Smith) A. DC. & Fagaceae & Dicot & Tree \\
\hline 76. & Celosia argentea $\mathrm{L}$. & Amarnthaceae & Dicot & Herb \\
\hline 77. & Centella asiatica $(\mathrm{L}$.$) Urban$ & Hydrocotylidaceae & Dicot & Herb \\
\hline 78. & Chickrassia tabularis (A. Juss.) Wight \& Am. & Meliaceae & Dicot & Tree \\
\hline 79. & Chrysopogon aciculatus (Retz.) Trin. & Poaceae & Dicot & Herb \\
\hline 80. & Cissus adnata Roxb. & Vitaceae & Dicot & Climber \\
\hline 81. & Cissus repens Lamk. & Vitaceae & Dicot & Climber \\
\hline 82. & Clausena heptaphylla (Roxb.) Wight \& Arn. Ex Steud. & Rutaceae & Dicot & Shrub \\
\hline 83. & Clerodendrum viscossum Vent. & Verbenaceae & Dicot & Shrub \\
\hline 84. & Coccinia cordifolia Cogn. & Cucurbitaceae & Dicot & Climber \\
\hline 85. & Coffea bengalensis Roxb. ex Schult. & Rubiaceae & Dicot & Shrub \\
\hline 86. & Colocasia esculenta (L.) Schott & Araceae & Monocot & Herb \\
\hline 87. & Combretum acuminatum Roxb. & Combretaceae & Dicot & Shrub \\
\hline 88. & Commelina benghalensis $\mathrm{L}$. & Commelinaceae & Monocot & Herb \\
\hline 89. & Costus speciosus (Koenig ex Retz.) Smith & Costaceae & Monocot & Herb \\
\hline 90. & Crataeva nurvala Buch.-Ham. & Cappridaceae & Dicot & Tree \\
\hline 91. & Crinum asiaticum $\mathrm{L}$. & Liliaceae & Monocot & Herb \\
\hline 92. & Crotalaria pallida Ait. & Fabaceae & Dicot & Herb \\
\hline 93. & Croton banplandianum Baill. & Euphorbiaceae & Dicot & Herb \\
\hline 94. & Curcuma amada Roxb. & Zingiberaceae & Monocot & Herb \\
\hline 95. & Cyathia gigantean (Wall. Ex Hook.) Holtt. & Cyathaceae & Ptidophyte & Tree \\
\hline 96. & Cyperus kyllinga Endl. & Cyperaceae & Monocot & Herb \\
\hline 97. & Dactyloctenium aegepticum (L.) P. Beauv. & Poaceae & Monocot & Herb \\
\hline 98. & Daemonorops jenkinsianus (Griff.) Martius & Arecaceae & Monocot & Climber \\
\hline 99. & Dalbergia sisoo Roxb. & Fabaceae & Dicot & Tree \\
\hline
\end{tabular}


Table 1. Continued...

\begin{tabular}{|c|c|c|c|c|}
\hline Sl. No. & Scientific name & Family & Cotyledon & Habit \\
\hline 100. & Dalbergia spinosa Roxb. & Fabaceae & Dicot & Shrub \\
\hline 101. & Dalbergia stipulacea Roxb. & Fabaceae & Dicot & Shrub \\
\hline 102. & Datura metel L. & Solanaceae & Dicot & Herb \\
\hline 103. & Delonix regia Rafin. & Fabaceae & Dicot & Tree \\
\hline 104. & Dentella repens (L.) J. R. \& G. Forst. & Rubiaceae & Dicot & Herb \\
\hline 105. & Derris elliptica (Wall.) Benth. & Fabaceae & Dicot & Climber \\
\hline 106. & Derris robusta (Roxb. ex DC.) Benth. & Fabaceae & Dicot & Climber \\
\hline 107. & Desmodium leguminosarum & Fabaceae & Dicot & Herb \\
\hline 108. & Desmodium triflorum (L.) DC. & Fabaceae & Dicot & Herb \\
\hline 109. & Desmos chinensis Lour. & Annonaceae & Dicot & Climber \\
\hline 110. & Dicranopteris linearis (Burm. f.) Underw. & Gleicheniaceae & Pteridophyte & Herb \\
\hline 111. & Dillenia indica $\mathrm{L}$. & Dilleniaceae & Dicot & Tree \\
\hline 112. & Dillenia pentagyna Roxb. & Dilleniaceae & Dicot & Tree \\
\hline 113. & Dioscorea alata $\mathrm{L}$. & Dioscoreaceae & Monocot & Climber \\
\hline 114. & Dioscorea belophylla (Prain) Voigt ex Haines & Dioscoreaceae & Monocot & Climber \\
\hline 115. & Dioscorea bulbifera $\mathrm{L}$. & Dioscoreaceae & Monocot & Climber \\
\hline 116. & Dioscorea pentaphylla L. & Dioscoreaceae & Monocot & Climber \\
\hline 117. & Diospyros peregrine Guerke & Ebenaceae & Dicot & Tree \\
\hline 118. & Diplazium esculentum (Retz.) Sw. & Athyriaceae & Pteridophyte & Herb \\
\hline 119. & Dipterocarpus turbinatus Roxb. & Dipterocarpaceae & Dicot & Tree \\
\hline 120. & Dracaena spicata Roxb. & Liliaceae & Monocot & Herb \\
\hline 121. & Duabanga grandiflora (Roxb. ex DC.) Walp. & Onneratiaceae & Dicot & Tree \\
\hline 122. & Eclipta alba (L.) Hassk. & Asteraceae & Dicot & Herb \\
\hline 123. & Elaeocarpus floribundus Blume & Elaeocarpaceae & Dicot & Tree \\
\hline 124. & Elaeocarpus robustus Roxb. & Elaeocarpaceae & Dicot & Tree \\
\hline 125. & Elephantopous scaber L. & Asteraceae & Dicot & Herb \\
\hline 126. & Elusine indica (L.) Gaertn. & Poaceae & Monocot & Herb \\
\hline 127. & Emilia sonchifolia (L.) DC. & Asteraceae & Dicot & Herb \\
\hline 128. & Entada phaseoloides (L.) Merr. & Fabaceae & Dicot & Climber \\
\hline 129. & Eragrostis tenella (L.) P. Beauv. ex Roem. \& Schult. & Poaceae & Monocot & Herb \\
\hline 130. & Erythrina ovalifolia Roxb. & Fabaceae & Dicot & Tree \\
\hline 131. & Erythrina variegata L. & Fabaceae & Dicot & Tree \\
\hline 132. & Eucalyptus camadulensis Dehnhardt & Myrtaceae & Dicot & Tree \\
\hline 133. & Eupatorium odoratum L. & Asteraceae & Dicot & Shrub \\
\hline 134. & Euphorbia hirta L. & Euphorbiaceae & Dicot & Herb \\
\hline 135. & Evolvulus numularius (L.) L. & Convolvulaceae & Dicot & Herb \\
\hline 136. & Ficus benghalensis L. & Moraceae & Dicot & Tree \\
\hline 137. & Ficus bnjamina $\mathrm{L}$. & Moraceae & Dicot & Tree \\
\hline 138. & Ficus hirta Vahl & Moraceae & Dicot & Tree \\
\hline 139. & Ficus hispida L. f. & Moraceae & Dicot & Tree \\
\hline 140. & Ficus racemosa $\mathrm{L}$. & Moraceae & Dicot & Tree \\
\hline 141. & Ficus religiosa $\mathrm{L}$. & Moraceae & Dicot & Tree \\
\hline 142. & Ficus semicordata Buch.-Ham. ex Smith & Moraceae & Dicot & Tree \\
\hline 143. & Fimbristylis dichotoma (L.) Vahl & Cyperaceae & Monocot & Herb \\
\hline 144. & Fimbristylis miliacea (L.) Vahl & Cyperaceae & Monocot & Herb \\
\hline 145. & Flacourtia indica (Burm. f.) Merr. & Flacourtiaceae & Dicot & Shrub \\
\hline 146. & Flacourtia jangomas (Lour.) Raeusch. & Flacourtiaceae & Dicot & Tree \\
\hline 147. & Flemingia bracteata (Roxb.) Wight & Fabaceae & Dicot & Shrub \\
\hline 148. & Floscopa scandans Lour. & Commelinaceae & Monocot & Herb \\
\hline 149. & Garcinia cowa Roxb. ex DC. & Cluceaceae & Dicot & Tree \\
\hline
\end{tabular}


Table 1. Continued...

\begin{tabular}{|c|c|c|c|c|}
\hline Sl. No. & Scientific name & Family & Cotyledon & Habit \\
\hline 150. & Garcinia xanthochymus Hook. f. ex T. Anders. & Cluceaceae & Dicot & Tree \\
\hline 151. & Globba marantina $\mathrm{L}$. & Zingiberaceae & Monocot & Herb \\
\hline 152. & Glochidion lanceolarium (Roxb.) Voigt & Euphorbiaceae & Dicot & Shrub \\
\hline 153. & Glochidion multiloculare (Roxb. ex Willd.) Muell.-Arg. & Euphorbiaceae & Dicot & Shrub \\
\hline 154. & Glycosmis pentaphylla (Retz.) A. DC. & Rutaceae & Dicot & Shrub \\
\hline 155. & Gmelina arborea Roxb. & Verbenaceae & Dicot & Tree \\
\hline 156. & Gnaphalium luteo-alba L. & Asteraceae & Dicot & Herb \\
\hline 157. & Grewia asiatica $\mathrm{L}$. & Tiliaceae & Dicot & Shrub \\
\hline 158. & Grewia serrulata DC. & Tiliaceae & Dicot & Shrub \\
\hline 159. & Guazuma tomentosa & Sterculiaceae & Dicot & Shrub \\
\hline 160. & Hedyotis scandens Roxb. & Rubiaceae & Dicot & Climber \\
\hline 161. & Heliotropium indicum $\mathrm{L}$. & Boraginaceae & Dicot & Herb \\
\hline 162. & Hemidesmus indicus (L.) R. Br. & Asclepiadaceae & Dicot & Climber \\
\hline 163. & Holarrhena antidysenterica (L.) Wall. ex Decne. & Asclepiadaceae & Dicot & Shrub \\
\hline 164. & Homalomena aromatica (Roxb. ex Sim) Schott. & Araceae & Monocot & Herb \\
\hline 165. & Hydnocarpus kurzii (King) Warb. & Flacourtiaceae & Dicot & Tree \\
\hline 166. & Hyptis suaveolens (L.) Poit. & Lamiaceae & Dicot & Herb \\
\hline 167. & Ichnocarpus frutescens (L.) R. Br. & Apocynaceae & Dicot & Climber \\
\hline 168. & Ilex godajam Colebr. & Aquifoliaceae & Dicot & Tree \\
\hline 169. & Imperata cylindrical (L.) P. Beav. & Poaceae & Monocot & Herb \\
\hline 170. & Ipomoea fistulosa Mart. Ex Choisy & Convolvulaceae & Dicot & Shrub \\
\hline 171. & Ixora arborea Roxb. ex Smith & Rubiaceae & Dicot & Shrub \\
\hline 172. & Ixora spectabilis Wall. Ex Don & Rubiaceae & Dicot & Shrub \\
\hline 173. & Ixora undulata Roxb. & Rubiaceae & Dicot & Shrub \\
\hline 174. & Jasminum multiflorum (Burm. f.) Andr. & Oleaceae & Dicot & Shrub \\
\hline 175. & Jasminum sambac Ait. & Oleaceae & Dicot & Shrub \\
\hline 176. & Justicia diffusa Willd. & Acanthaceae & Dicot & Herb \\
\hline 177. & Lagerstroemia speciosa L. Pers. & Lythraceae & Dicot & Tree \\
\hline 178. & Lannea coromandelica (Houtt.) Merr. & Anacardiaceae & Dicot & Tree \\
\hline 179. & Lantana camara L. & Verbenaceae & Dicot & Shrub \\
\hline 180. & Laportea crenulata Gaud. & Urticaceae & Dicot & Climber \\
\hline 181. & Leea acuminate (Burm. f.) Merr. & Leaceae & Dicot & Shrub \\
\hline 182. & Leea robusta Roxb. & Lea & Dicot & Shrub \\
\hline 183. & Leucaena leucocephala (Lamk.) DC. Wit. & Fabaceae & Dicot & Tree \\
\hline 184. & Leucas aspera (Willd.) Link. & Lamiaceae & Dicot & Herb \\
\hline 185. & Leucas lavendulifolia Smith. & Lamiaceae & Dicot & Herb \\
\hline 186. & Lindernia procumbens (Krocker) Philcox & Scrophulariaceae & Dicot & Herb \\
\hline 187. & Lippia nodiflora (L.) Rich. & Verbenaceae & Dicot & Herb \\
\hline 188. & Litsea monopetala (Roxb.) Pers. & Lauraceae & Dicot & Tree \\
\hline 189. & Lycopodium $\mathrm{sp}$. & Lycopodiaceae & Pteridophyte & Climber \\
\hline 190. & Lygodium flexuosum (L.) Sw. & LygodIaceae & Pteridophyte & Climber \\
\hline 191. & Macaranga denticulate (Blume) Muell.-Arg. & Euphorbiaceae & Dicot & Tree \\
\hline 192. & Maesa montana A. DC. & Myrsinaceae & Dicot & Shrub \\
\hline 193. & Madhuca indica (L.) Rich. & Sapotaceae & Dicot & Tree \\
\hline 194. & Mallotus philippnsis (Lamk.) Muell.-Arg. & Euphorbiaceae & Dicot & Shrub \\
\hline 195. & Mangifera indica $\mathrm{L}$. & Anacardiaceae & Dicot & Tree \\
\hline 196. & Mangifera sylvatica Roxb. & Anacardiaceae & Dicot & Tree \\
\hline 197. & Manihot esculenta Crantz. & Euphorbiaceae & Dicot & Tree \\
\hline 198. & Melastoma malabathrium Roxb. & Melastomaceae & Dicot & Shrub \\
\hline 199. & Melia sempervirens (L.) sw. & Meliaceae & Dicot & Tree \\
\hline
\end{tabular}


Table 1. Continued...

\begin{tabular}{|c|c|c|c|c|}
\hline Sl. No. & Scientific name & Family & Cotyledon & Habit \\
\hline 200. & Melilotus alba Desr. & Fabaceae & Dicot & Shrub \\
\hline 201. & Merremia hirta (L.) Merr. & Convolvulaceae & Dicot & Climber \\
\hline 202. & Mesua ferrea $\mathrm{L}$. & Clauciaceae & Dicot & Tree \\
\hline 203. & Microcos paniculata $\mathrm{L}$. & Tiliaceae & Dicot & Shrub \\
\hline 204. & Micromelum minutum (Forst. f.) Wight. \& Arn. & Rutaceae & Dicot & Shrub \\
\hline 205. & Mikania cordata (Burm. f.) B. L. Roxb. & Asteraceae & Dicot & Climber \\
\hline 206. & Mimosa pudica L. & Fabaceae & Dicot & Herb \\
\hline 207. & Mimusops elengi $\mathrm{L}$ & Sapotaceae & Dicot & Tree \\
\hline 208. & Moghania latifolia (Benth.) Mukherjee & Fabaceae & Dicot & Shrub \\
\hline 209. & Morinda angustifolia Roxb. & Rubiaceae & Dicot & Shrub \\
\hline 210. & Mucuna imbricata (DC.) Baker & Fabaceae & Dicot & Climber \\
\hline 211. & Mucuna pruriens (L.) DC. & Fabaceae & Dicot & Climber \\
\hline 212. & Murdannia nudiflora (L.) Brenan & Commelinaceae & Monocot & Herb \\
\hline 213. & Musa ornata $\operatorname{Roxb}$ & Musaceae & Monocot & Herb \\
\hline 214. & Mussanda roxburghii Hook. f. & Rubiaceae & Dicot & Shrub \\
\hline 215. & Mycetia longifolia (Wall.) O. Kuntze. & Rubiaceae & Dicot & Shrub \\
\hline 216. & Nelsonia canescens (Lamk.) Spreng. & Acanthaceae & Dicot & Herb \\
\hline 217. & Nerium indicum Mill. & Apocynaceae & Dicot & Tree \\
\hline 218. & Ocimum sanctum $\mathrm{L}$. & Lamiaceae & Dicot & Herb \\
\hline 219. & Oldenlandia corymbosa $\mathrm{L}$. & Rubiaceae & Dicot & Herb \\
\hline 220. & Ophiorrhiza mugna $\mathrm{L}$. & Rubiaceae & Dicot & Herb \\
\hline 221. & Oroxylum indicum (L.) Kurz. & Bignoniaceae & Dicot & Tree \\
\hline 222. & Oxalis corniculata $\mathrm{L}$. & Oxalidaceae & Dicot & Herb \\
\hline 223. & Paederia foetida $\mathrm{L}$. & Rubiaceae & Dicot & Climber \\
\hline 224. & Pandanus foetidus Roxb. & Pandanaceae & Monocot & Shrub \\
\hline 225. & Panicum repens $\mathrm{L}$. & Poaceae & Monocot & Herb \\
\hline 226. & Passiflora foetida $\mathrm{L}$. & Passifloraceae & Dicot & Herb \\
\hline 227. & Peperomia pellucida (L.) H. B. \& K. & Piperaceae & Dicot & Herb \\
\hline 228. & Peristrophe bicalyculata (Retz.) Nees & Acanthaceae & Dicot & Herb \\
\hline 229. & Persicaria hydropiper (L.) Spach & Polygonaceae & Dicot & Herb \\
\hline 230 . & Persicaria orientalis (L.) Spach & Polygonaceae & Dicot & Herb \\
\hline 231. & Phlogacanthus thyrsiflorus Nees. & Acanthaceae & Dicot & Shrub \\
\hline 232. & Phyllanthus embelica $\mathrm{L}$. & Euphorbiaceae & Dicot & Tree \\
\hline 233. & Phyllanthus niruri $\mathrm{L}$. & Euphorbiaceae & Dicot & Herb \\
\hline 234. & Phyllanthus reticulatus Poir & Euphorbiaceae & Dicot & Shrub \\
\hline 235. & Physalis minima $\mathrm{L}$. & Solanaceae & Dicot & Herb \\
\hline 236. & Piper longum L. & Piperaceae & Dicot & Climber \\
\hline 237. & Piper sylvaticum Roxb. & Piperaceae & Dicot & Climber \\
\hline 238. & Polyalthia longifolia (Sonn.) Thw. & Annonaceae & Dicot & Tree \\
\hline 239. & Premna corymbosa Rott. \& Willd. & Verbenaceae & Dicot & Shrub \\
\hline 240. & Premna esculenta Roxb. & Verbenaceae & Dicot & Shrub \\
\hline 241. & Proniferium nudatum (Roxb. ex Griff.) Holtt. & Thelypteridaceae & Pteridophyte & Herb \\
\hline 242. & Psidium guajava $\mathrm{L}$ & Myrtaceae & Dicot & Tree \\
\hline 243. & Pterygota alata (Roxb.) R. Br. & Sterculiaceae & Dicot & Tree \\
\hline 244. & Pteris vittata $\mathrm{L}$ & Pteridaceae & Pteridophyte & Herb \\
\hline 245. & Pterospermum acerifolium (L.) Willd. & Sterculiaceae & Dicot & Tree \\
\hline 246. & Pterospermum semisegittatum Buch.-Ham. ex Roxb. & Sterculiaceae & Dicot & Tree \\
\hline 247. & Quercus spicata Smith & Fagaceae & Dicot & Tree \\
\hline 248. & Rauwolfia sarpentina (L.) Benth. ex Kurz. & Apocynaceae & Dicot & Herb \\
\hline 249. & Ricinus communis $\mathrm{L}$. & Euphorbiaceae & Dicot & Shrub \\
\hline
\end{tabular}


Table 1. Continued...

\begin{tabular}{|c|c|c|c|c|}
\hline Sl. No. & Scientific name & Family & Cotyledon & Habit \\
\hline 250. & Rorippa indica (L.) Hiern & Brassicaceae & Dicot & Herb \\
\hline 251. & Ruellia tuberosa L. & Acanthaceae & Dicot & Herb \\
\hline 252. & Saccharum spontaneum L. & Poaceae & Monocot & Herb \\
\hline 253. & Scleria levis Retz. & Cyperaceae & Monocot & Herb \\
\hline 254. & Scoparia dulcis L. & Scrophulariaceae & Dicot & Herb \\
\hline 255. & Selaginella vaginata Spring & Selaginellaceae & Pteridophyte & Herb \\
\hline 256. & Shorea robusta Gaertn. & Dipterocarpaceae & Dicot & Tree \\
\hline 257. & Sida acuta Burm. f. & Malvaceae & Dicot & Shrub \\
\hline 258. & Sida cordata (Burm. f.) Borss. & Malvaceae & Dicot & Herb \\
\hline 259. & Sida cordifolia $\mathrm{L}$. & Malvaceae & Dicot & Herb \\
\hline 260. & Smilax prolifera Roxb. & Smilacaceae & Monocot & Climber \\
\hline 261. & Smilax zylanica $\mathrm{L}$. & Smilacaceae & Monocot & Climber \\
\hline 262. & Solanum indicum $\mathrm{L}$. & Solanaceae & Dicot & Shrub \\
\hline 263. & Solanum nigram L. & Solanaceae & Dicot & Herb \\
\hline 264. & Solanum torvum Swartz & Solanaceae & Dicot & Shrub \\
\hline 265. & Spatholobus roxburghii Benth. & Fabaceae & Dicot & Climber \\
\hline 266. & Spondias pinnata (L. f.) Kurz. & Acanthaceae & Dicot & Tree \\
\hline 267. & Stemona tuberosa Lour. & Stemonaceae & Monocot & Climber \\
\hline 268. & Stephania japonica (Thunb.) Miers & Menispermaceae & Dicot & Climber \\
\hline 269. & Sterculia vilosa Roxb. & Sterculiaceae & Dicot & Tree \\
\hline 270. & Steudnera colocasioides Hook. f. & Araceae & Monocot & Herb \\
\hline 271. & Streblus asper Lour. & Moraceae & Dicot & Tree \\
\hline 272. & Suregada multiflora (A. Juss.) Baill. & Euphorbiaceae & Dicot & Shrub \\
\hline 273. & Swietenia mahagoni Jacq. & Meliaceae & Dicot & Tree \\
\hline 274. & Swintonia floribunda Griff. & Anacardiaceae & Dicot & Tree \\
\hline 275. & Synedrella nodiflora (L.) Gaertn. & Asteraceae & Dicot & Herb \\
\hline 276. & Syzygium cumini (L.) Skeels & Myrtaceae & Dicot & Tree \\
\hline 277. & Syzygium fruticosa DC. & Myrtaceae & Dicot & Tree \\
\hline 278. & Syzygium operculatum (Roxb.) Niedz. & Myrtaceae & Dicot & Tree \\
\hline 279. & Tabernaemontana recurva Roxb. & Apocynaceae & Dicot & Shrub \\
\hline 280. & Tamarindus indica $\mathrm{L}$. & Fabaceae & Dicot & Tree \\
\hline 281. & Tectona grandis L. & Verbenaceae & Dicot & Tree \\
\hline 282. & Tephrosia purpurea (L.) Pers. & Fabaceae & Dicot & Shrub \\
\hline 283. & Terminalia arjuna (Roxb. ex DC.) Wight \& Arn. & Combretaceae & Dicot & Tree \\
\hline 284. & Terminalia belerica (Gaertn.) Roxb. & Combretaceae & Dicot & Tree \\
\hline 285. & Terminalia capata $\mathrm{L}$. & Combretaceae & Dicot & Tree \\
\hline 286. & Terminalia chebula Retz. & Combretaceae & Dicot & Tree \\
\hline 287. & Tetrastigma agustifolium (Roxb.) Planch. & Vitaceae & Dicot & Climber \\
\hline 288. & Thunbergia grandiflora (Roxb. ex Rottler) Roxb. & Acanthaceae & Dicot & Climber \\
\hline 289. & Thysanolaena maxima (Roxb.) O. Kuntze & Poaceae & Monocot & Herb \\
\hline 290. & Tinospora crispa (L.) Hook. f. \& Thorns. & Menispermaceae & Dicot & Climber \\
\hline 291. & Trema orientalis (L.) Blume & Ulmaceae & Dicot & Tree \\
\hline 292. & Trewia nodiflora $\mathrm{L}$. & Euphorbiaceae & Dicot & Tree \\
\hline 293. & Tridex procumbens $\mathrm{L}$. & Asteraceae & Dicot & Herb \\
\hline 294. & Tylophora indica (Burm. f.) Merr. & Asclepiadaceae & Dicot & Climber \\
\hline 295. & Typhonium trilobatum (L.) Schott. & Araceae & Monocot & Herb \\
\hline 296. & Urena lobata $\mathrm{L}$ & Malvaceae & Dicot & Shrub \\
\hline 297. & Urena sinuata $\mathrm{L}$. & Malvaceae & Dicot & Shrub \\
\hline 298. & Vernonia cinerea (L.) Less. & Asteraceae & Dicot & Herb \\
\hline 299. & Vetiveria zizaniodes (L.) Nash & Poaceae & Monocot & Herb \\
\hline
\end{tabular}


Table 1. Continued...

\begin{tabular}{|c|c|c|c|c|}
\hline Sl. No. & Scientific name & Family & Cotyledon & Habit \\
\hline 300. & Vitex nigundo L. & Verbenaceae & Dicot & Shrub \\
\hline 301. & Vitis trifolia L. & Vitaceae & Dicot & Climber \\
\hline 302. & Willoughdeia edulis Roxb. & Apocynaceae & Dicot & Climber \\
\hline 303. & Xanthium indicum Koen. ex Roxb. & Asteraceae & Dicot & Shrub \\
\hline 304. & Xeromphis spinosa (Thunb.) Keay & Rubiaceae & Dicot & Shrub \\
\hline 305. & Xylia dolabiformis Benth. & Fabaceae & Dicot & Tree \\
\hline 306. & Zanthoxyllum rhetsa (Roxb.) DC. & Rutaceae & Dicot & Tree \\
\hline 307. & Ziziphus mauritiana Lamk. & Rhamnaceae & Dicot & Tree \\
\hline 308. & Ziziphus oenoplia (L.) Mill. & Rhamnaceae & Dicot & Shrub \\
\hline 309. & Ziziphus rugosa Lamk. & Rhamnaceae & Dicot & Shrub \\
\hline
\end{tabular}

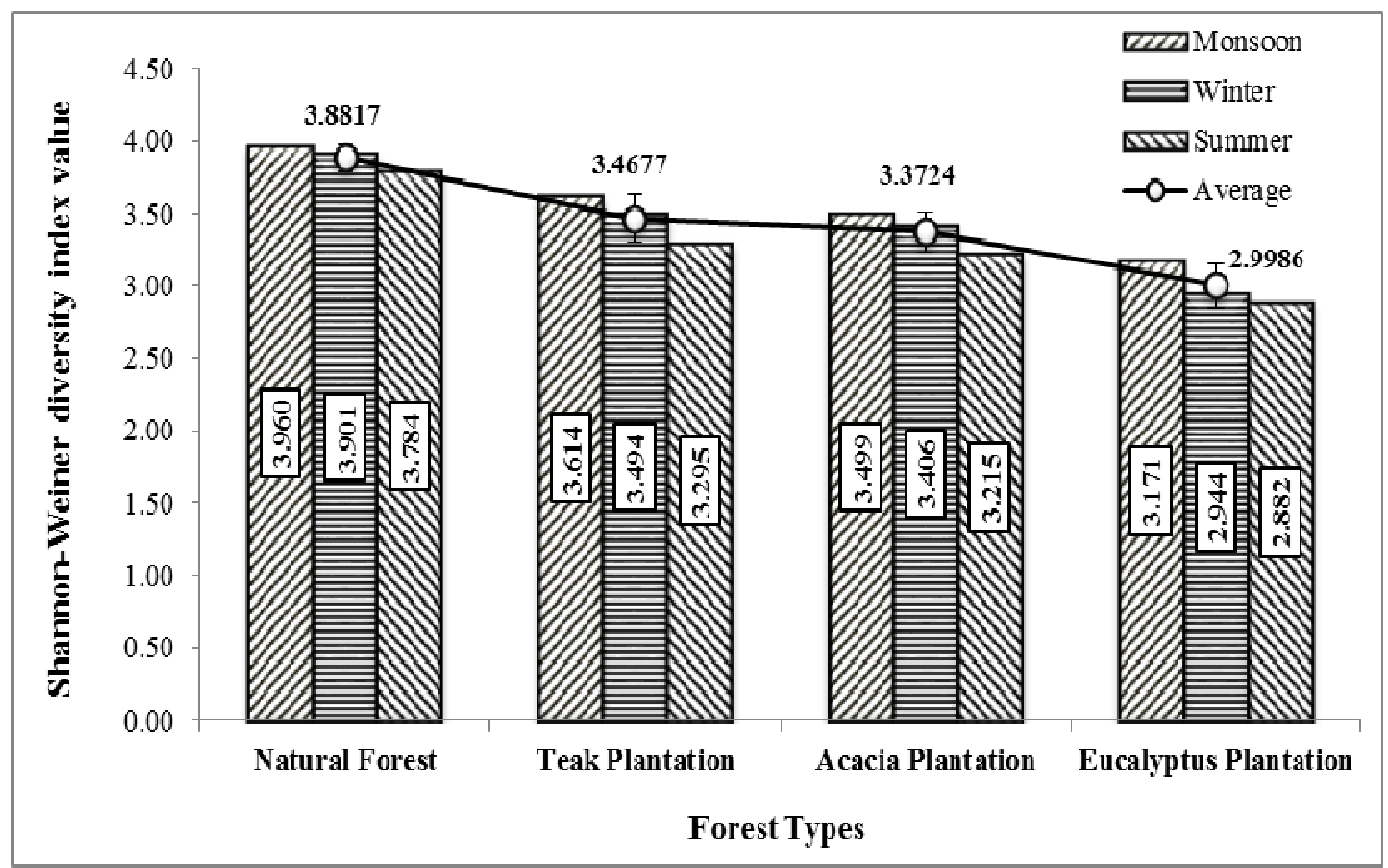

Fig. 5. Shannon-Weiner diversity index values recorded from different selected forest sites of Rema-Kalenga wildlife sanctuary

In plantation forest, the highest number of 194 plant species under 178 genera and 67 families were recorded from Tectona plantation site during monsoon season, which was followed by 164 plant species under 131 genera and 63 families were recorded from Acacia plantation site during the same season, whereas the lowest number of 105 plant species belonging to 86 genera and 45 families were recorded from Eucalyptus plantation site during summer season (Table 2). In Tectona plantation forest, the highest ShannonWiener index value 3.614 was calculated during monsoon season which was followed by 3.494 and 3.215 found during winter and summer seasons, respectively (Table 2; Fig. 5). In Acacia plantation forest, the highest index value 3.496 was recorded during monsoon 
season which was followed by 3.406 and 3.291 recorded during winter and summer seasons, respectively. Similarly, In Eucalyptus plantation forest, the highest index value 3.171 was recorded during monsoon season which was followed by 2.944 and 2.882 were recorded during winter and summer seasons, respectively (Table 2; Fig. 5).

Table 2. Floristic composition and Shannon-Weiner diversity index values recorded from four different types of forest sites of Rema-Kalenga wildlife sanctuary

\begin{tabular}{l|l|ccc|c}
\hline \multirow{2}{*}{ Name of the Site } & Season & \multicolumn{3}{|c|}{ Floristic composition } & \multirow{2}{*}{$\begin{array}{c}\text { Diversity } \\
\text { Index }\end{array}$} \\
\cline { 3 - 5 } Natural & & No. of family & No. of genera & No. of species & 3.960 \\
forest & Monsoon & 79 & 237 & 298 & 3.901 \\
& Winter & 75 & 234 & 287 & 3.784 \\
& Summer & 69 & 221 & 245 & - \\
& Total & 81 & 240 & 304 & $3.88 \pm 0.090$ \\
\hline Tectona & Average & 74.33 & 197.33 & 276.67 & 3.614 \\
plantation & Monsoon & 67 & 178 & 194 & 3.494 \\
& Winter & 59 & 136 & 159 & 3.215 \\
& Summer & 52 & 94 & 124 & - \\
& Total & 69 & 180 & 197 & $3.441 \pm 0.205$ \\
\hline Acacia & Average & 59.33 & 136.00 & 159.00 & 3.496 \\
plantation & Monsoon & 63 & 131 & 164 & 3.406 \\
& Winter & 56 & 127 & 152 & 3.291 \\
& Summer & 49 & 115 & 130 & - \\
& Total & 64 & 131 & 165 & $3.398 \pm 0.103$ \\
\hline Eucalyptus & Average & 56.00 & 124.33 & 148.67 & 3.171 \\
plantation & Monsoon & 52 & 119 & 138 & 2.944 \\
& Winter & 48 & 103 & 126 & 2.882 \\
& Summer & 45 & 86 & 105 & - \\
& Total & 52 & 119 & 139 & $2.999 \pm 0.152$ \\
\hline & Average & 48.33 & 102.67 & 123.00 & \\
\hline & & & & &
\end{tabular}

The average number of taxa and their diversity index values recorded from four different types of forest sites during three different seasons were found to decrease by different sites of plantation forests in the sequence from Natural forest to Tectona plantation to Acacia plantation to Eucalyptus plantation forest. Similarly, the number of plant taxa and Shannon-Wiener diversity index values varied among the studied seasons and found to show the trends of gradual decrease from monsoon to winter to summer seasons.

The number of undergrowth plant taxa as well as the phytodiversity index values was found to be the highest in natural forest site which was followed by Tectona and Acacia plantation sites and the lowest in Eucalyptus plantation site (Fig. 2 and Fig. 5). The finding of lower number of undergrowth species in the plantation of fast-growing exotic tree species is supported by that of Hossain et al. (2002) The occurrence of lower number of undergrowth species in the plantation forest of fast-growing exotic tree species 
(Hossain et al., 2002) that due to thick, leathery, flattened and expanded leaves of exotic plants were degrade slowly and ultimately the soil of their habitat become hard and unfertile. The finding of natural forests harboring the maximum species diversity index in their comparatively stable ecosystem in respect to any other plantation forest ecosystem is supported by Narayan et al. (1994) because they stated that higher values of diversity showed greater stability of the community. Eucalyptus plantation depressed the forest species more than other plantation either fast-growing Acacia or slow-growing Tectona plantation. Due to allelopathy Eucalyptus plantation is often regarded as damaging for the environment (Poore \& Fries, 1985) which was also shown by Lisanework \& Michelsen (1993), Souto et al. (1995), Bernhard-Reversat (1999), Reigosa et al. (1999). Moreover, the floristic composition and phytodiversity of fast-growing plantation forests in tropical regions have been reduced through the losing of fertility of top soil. The finding of Acacia and Eucalyptus plantations sites of the study area harboring fewer indigenous plant species and less species diversity is supported by the findings of Hossain \& Hossain (2009), Bouvet (1998), Abbasi \& Vinithan (1997).

The present findings concluded that any kind of plantations, either of fast-growing Acacia or Eucalyptus or slow-growing Tectona species in this semi-tropical region might affect the species composition and diversity of local forests. Fast-growing exotic tree plantations of Acacia and Eucalyptus affected the floristic composition and phytodiversity as well as on the environment more than that of Tectona plantation. Therefore, it might be suggested that monoculture plantations especially for the fast-growing exotic species like Acacia, Eucalyptus are not suitable for local forest plant species in the semi-tropical region of Rema-Kalenga wildlife sanctuary. These types of fast-growing species may be selected for plantation in unfertile barren lands or less fertile grasslands, because they grow well in those types of habitats without any care and supplementary nourishment (Hossain et al., 2002). So, the fast-growing exotic tree plantation of Acacia and Eucalyptus should be avoided for future sustainable management and development of Rema-Kalenga wildlife sanctuary.

\section{REFERENCES}

Abbasi, S.A. and Vinithan, S. 1997. Ecological impacts of eucalyptus - myths and realities. Indian Forester. 123: 710-739.

Ahmed, Z.U., Begum, Z.N.T., Hassan, M.A., Khondker, M.M., Kabir, S.M.H., Ahmad, M., Ahmed, A.T.A., Rahman,A .K.A. and Haque, E.U. (eds.). 2008-2009. Encyclopedia of Flora and Fauna of Bangladesh. 6-10. Angiosperms; Dicotyledons. Asiatic Society of Bangladesh, Dhaka.

Alexiades, M.N. 1996. Selected Guidelines for Ethno botanical Research: A Field Manual. The New York Botanical Garden, New York. pp. 99-133.

Bernhard-Reversat, F. 1999. The leaching of Eucalyptus hybrids and Acacia auriculiformis leaf litter: laboratory decomposition of tropical leaf litters. Europian J. Soil Biology. 34: 117122. 
Bouillet, J.P. and Bernhard-Revesat, F. 2001. General objectives and sites. In: Bernhard-Revesat, F. (ed.). Effect of exotic tree plantations on plant diversity and biological soil fertility in the Congo Savanna: with special reference to Eucalyptus. Center for International Forestry Research, Bogor, Indonesia. p. 71.

Bouvet, J.M. 1998. Les plantation d'Eucalyptus. Evolutions recentes et perspectives. Internal Note. CIRAD-Foret, Montpellier, France, p. 35.

Braun-Blanquet, J. 1932. Plant sociology: The study of plant communities. McGeaw-Hill Book Co. Inc. New York.

Canonizado, J.A. and Rahman, S.M. 1998. GBO/WB Forest resources management project technical assistance component: integrated forest management plan for the Sylhet forest division. Mandala Agricultural Development corporation and Forest Department, Ministry of Environment and Forest, Dhaka, Bangladesh, pp. 1-4.

Champion, H.G. 1936. A Preliminary Survey of the Forest Types of India and Burma. Indian Forest Records. 1: 1.

Evans, J. 1992. Plantation forestry in tropics. $2^{\text {nd }}$ edn., Oxford University Press, Oxford. pp. 403.

FAO. 1988. Land Resources of Bangladesh for Agricultural Development. Agrological Regions of Bangladesh. BGD/81/035 Technical Report 2, p. 570.

FAO. 1993. Forest resources assessment 1990. Tropical countries. FAO Forestry Paper 112. FAO, Rome, p. 61.

Gauthier, J.J. 1991. Les bois de plantation dans le commerce mondial des produits forestiers. In: L'emergence des nouveaux potentiels forestiers dans le monde. AFOCEL, Paris, pp. 9-20.

Green. 1990. IUCN Directory of South Asian protected areas. IUCN-The World Conservation Union, Gland, Switzerland and Cambridge, UK. pp. 24-30.

Hooker, J.D. 1872-1897. The Flora of British India. Vol. I-VII. Second edn. Periodical Expert Book Agency, Delhi, India. pp. 5568.

Hossain, A.B.M.E., Ahmed, I.J., Khan, S.A. and Hossain, G.M. 2002. Effect on local undergrowth biodiversity due to monoculture plantation of Acacia auriculiformis. J. Asiatic Soc. Bangladesh, Sci. 28(1): 1-10.

Hossain, G.H. 2006. Environmental impact assessment of afforestation programs of the department of forestry in Sylhet forest division. M. Phil dissertation, submitted to Jahangirnagar University, Savar, Dhaka, Bangladesh. pp. 145.

Hossain, G.M. and Hossain, A.B.M.E. 2009. Effect of exotic tree plantations on soil quality and microbial load in the forest of Rema-Kalenga wildlife sanctuary. Bangladesh J. Life Sci. 21(2): 121-127.

Hossain, M.K. and Pasha, M.K. 2001. Alien Invasive Plants in Bangladesh and their Impacts on the Ecosystem. Assessment and Management of Alien Species that Threatened Ecosystem: Habitats and Species (ed. by Secretariat of the Convention on Biological Diversity), pp. 73-75. CBD, Technical Paper No. 21, Montreal, Canada.

Hyland, B.P.M. 1972. A technique for collecting botanical specimens in rain forest. Flora Malesiana Bulletin. 26: 2038-2040.

Islam, M.M., Amin, A.S.R. and Sarker, S.K. 2003. Bangladesh: Country Report. Invasive Alien Species in South-Southeast Asia: National Reports \& Directory of Resources (ed. by N Pallewatta, JK Reaser, AT Gutierrez), pp. 7-20. The Global Invasive Species Programme, Cape Town, South Africa.

Jain, S.K. and Raw, R.R. 1977. A handbook of field and herbarium methods. Today and Tomorrows Printers and Publishers. p.157.

Kanowski, P.J. and Savill, P.S. 1992. Forest plantations: towards sustainable practice. Chapter 6 in: C Sargent and S Bass (Eds). Plantation politics: forest plantations in development. Earthscan, London. 121-151. 
Kent, M. and Coker, P. 1992. Vegetation description and analysis: A practical approach. The CRC Press, Inc. Belhaven Press, London. pp. 363.

Lisanework, N. and Michelsen, A. 1993. Allelopathy in agroforestry systems: the effects of leaf extracts of Cupressus lusitanica and three Eucalyptus spp. On four Ethiopian crops. Agroforestry. 21: 63-74.

Narayan, D., Agarwal, M., Pandey, J. and Singh, J. 1994. Changes in vegetation characters downward of an aluminium factory in India. Ann. Bot. 73: 557-565.

Nishat, A., Huq, S.M.I., Barun, S.P., Reza, A.H.M.A. and Khan, A.S. M. (eds.). 2002. Bioecological zones of Bangladesh. IUCN Bangladesh counter office, p. 141.

Pandy, D. 1995. Forest resources assessment 1990. Tropical forest plantation resources. FAO Forestry Paper 128, p. 81.

Poore, M.E.D. and Fries, C. 1985. The ecological effect of Eucalyptus. F.A.O. Forestry Paper 59. FAO, Rome, p. 87.

Prain, D. 1903. Bengal Plants. Vols. 1\&2 (Ind. Repr. 1963). Botanical Survey of India, Calcutta. pp. 1-1319.

Prasad, R. 1988. Rehabilitation and revegetation of mined-out areas in Madhya Pradesh: some critical issues. J. Trop. For. 4(I \& II): 139-157.

Raunkiaer, C. 1934. The life-form of plants and statistical plant geography. Claredon Press, Oxford, pp. 632.

Reigosa, M.J., Sanchez-Moreiras, A. and Gonzales, L. 1999. Ecophysiological approach in allelopathy. Critical Review in Plant Science. 18: 577-608.

Rizvi, S.N.H. 1970. East Pakistan District Gazetteers for Sylhet. Government of East Pakistan Survices and General Administration Department, Dhaka. pp. 5-6.

Siddiqui, K.U., Islam, M.A., Ahmed, Z.U., Begum, Z.N.T., Hassan, M.A., Khondker, M.AM., Rahman, M.M., Kabir, S.M.H., Ahmad, M., Ahmed, A.T.A., Rahman,A .K.A. and Haque, E.U. (eds.). 2007. Encyclopedia of Flora and Fauna of Bangladesh. 11. Angiosperms; Monocotyledons. Asiatic Society of Bangladesh, Dhaka.

Souto, X.C., Gonzalez, I. and Reigosa, M.J. 1995. Allelopathy in forest environment in Galicia, NW Spain. Allelopathy Journal. 2: 67-68.

Uddin, M.Z. and Hassan, M.A. 2004. Flora of Rema-Kalenga Wildlife Sanctuary. IUCN Bangladesh Country Office, Dhaka, Bangladesh. pp.122.

Uddin, M.Z., Khan, M.S. and Hassan, M.A. 2001. Ethnobotanical plant records of Kalenga forest range (Habigonj), Bangladesh for malaria, jaundice, diarrhoea and dysentery. Bangladesh J. Plant Taxon. 8(1): 101-104. 